\title{
Giải tích ngẫu nhiên và ứng dụng trong thị trường tài chính
}

\author{
Vũ Đức Thắng
}

November 20, 2014

TRƯỜNG ĐAII HOQC KHOA HOC TỰ NHIÊN

ĐẠI HỌC QUỐC GIA HÀ NộI

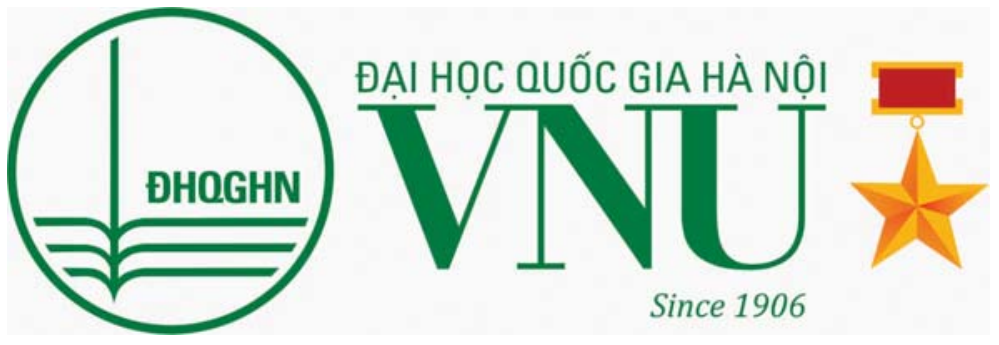

https://repository.vnu.edu.vn/handle/VNU_123/39531 


\section{TİM THEO}

Bản đồ thống kê

Đơn vị \& Bộ sưu tập (/community-

list)

Năm xuất bản

Giải tích ngẫu nhiên và ứng dụng trong thị trường tài chính : Luận văn ThS. Toán học: 60460106

(/browse?type=dateissueftł://repository.vnu.edu.vn/handle/VNU_123/39531 (http://repository.vnu.edu.vn/handle/VNU_123/39531) order=DESC)

Tác giả

(/browse?type=author)

Nhan đề

(/browse?type=title)

Chủ đề

(/browse?type=subject)

\section{XEM MÔ TẢ}

\section{HÒ SƠ TÁC}

14

GIẢ

(/BrowseAuthorProfile?ty be=auth pr)

\section{Nguyễn Kim Sơn \\ XEM \& TẢI}

(/BrowseAuthorProfile?type=author\&

order=DESC \&

sort_by $=2 \&$

$\mathrm{rpp}=10 \&$

value $=$ Nguy

$\% \mathrm{E} 1 \% \mathrm{BB}$

$\% 85 n \% 2 C+K i m+S$

\%C6\%A1n )(118)

Đinh Văn Toàn

\section{Tóm tắt}

62 tr. + CD-ROMLuận văn ThS. Lý thuyết xác suất và thống kê toán học -- Trường Đại học Khoa học Tự nhiên. Đại học Quốc gia Hà Nội, 2014Electronic Resources

\section{Nhà xuất bản}

ĐHKHTN

Trong bộ sưu tập: HUS - Master Theses (/handle/VNU_123/33316)

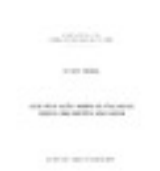

Xem trực tuyến (/flowpaper

$/$ ? bitsid=16ff39feea06-4775-99db$4 \mathrm{e} 16 \mathrm{c} 94111 \mathrm{fe})$

Yêu cầu tài liệu (/request-

item?handle=VNU_123 /39531\&

bitstream-

id $=16 \mathrm{ff} 39 \mathrm{fe}$ ea06-4775-99db$4 \mathrm{e} 16 \mathrm{c} 94111 \mathrm{fe})$
XEM MÔ TẢ XEM \& TẢI

107738985346698

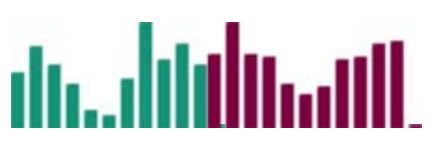

Thống kê nội dung

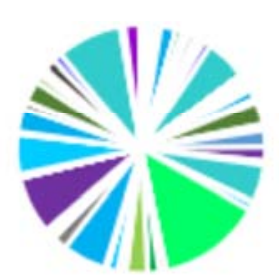


(/BrowseAuthorProfile?

$\begin{array}{ll}\text { order=DESC \& } & \text { value=V\%C5\%A9 } \\ \text { sort_by=2\& } & \% 2 \mathrm{C}+\% \mathrm{C} 4 \% 90 \% \mathrm{E} 1 \\ \text { rpp=10\& } & \% \mathrm{BB} \% \mathrm{~A} 9 \mathrm{C}+\mathrm{Th} \% \mathrm{E} 1 \% \mathrm{BA}\end{array}$

value $=$ Dinh $\% 2 C+$ Van+\% $\%$ Fanng)

)(67)

Người hướng dẫn

Nguyễn Hoàng Phan, Viết Thư, người

hướng dẫn

(/BrowseAuthorProfile?tbrowse?type=author\&

value=Phan $\% 2 \mathrm{C}+\mathrm{V}$

$\%$ E1\%BA\%BFt+Th

$\% \mathrm{C} 6 \% \mathrm{~B} 0 \% 2 \mathrm{C}+\mathrm{ng}$

$\% \mathrm{C} 6 \% \mathrm{~B} 0 \% \mathrm{E} 1 \% \mathrm{BB}$

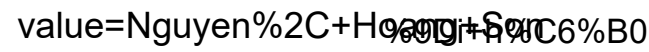

)(51) $\quad \% E 1 \% B B \% 9 B n g+d$

Nguyễn Huy

$\% \mathrm{E} 1 \% \mathrm{BA} \% \mathrm{ABn}$ )

Chương Xem chi tiết metadata

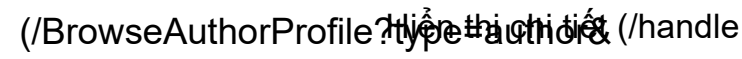

order=DESC\& INN_123

sort_by $=2 \& \quad$ /39531? mode=full)

$\mathrm{rpp}=10$ \&

value $=\mathrm{Nguy}$

$\% \mathrm{E} 1 \% \mathrm{BB}$

Thống kê truy cập (/handle/VNU_123/39531/statistics)

$\% 85 n \% 2 \mathrm{C}+\mathrm{Huy}+\mathrm{Ch}$

$\% \mathrm{C} 6 \% \mathrm{~B} 0$

$\% \mathrm{C} 6 \% \mathrm{~A} 1 \mathrm{ng})(48)$

Nguyễn Hoàng

Hải

(/BrowseAuthorProfile?type=author\&

order=DESC\&

sort_by $=2 \&$ 


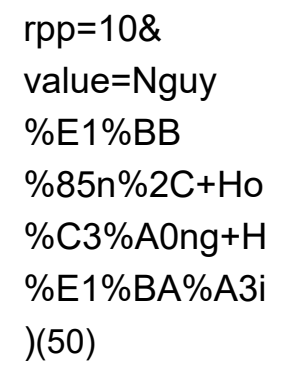

\begin{tabular}{l|l|l} 
Giới Thiệu Chính sách FAQ & Fa
\end{tabular}

Find us on

(http://dlcorp.com.vn/) 


\section{TÀI LIỆU THAM KHẢO:}

1. Dung, N. T. (2014). Một số tìm hiểu tiếp theo về bổ túc xác suất. Luận văn Thạc sĩ Khoa học Toán học, Trường Đại học Khoa học Tự nhiên, Đại học Quốc gia Hà Nội.

2. Hữu, N. V., \& Hoàng, V. Q. (2007). Các phương pháp toán học trong tài chính. NXB Đại học Quốc gia, Hà Nội.

3. Nguyên, T. T. (2011). Cơ sở toán tài chính. NXB Khoa học và Kỹ thuật, Hà Nội.

4. Thao, T. T. (2013). Toán tài chính căn bản. NXB Khoa học và Kỹ thuật, Hà Nội.

5. Thao, T. H.. (2000). Tích phân ngẫu nhiên \& Phuong trình vi phân ngẫu nhiên. NXB Khoa học và Ky thuật, Hà Nội.

6. Thao, T. H. (2009). Nhập môn Toán học Tài chính. NXB Khoa học và Kỹ thuật, Hà Nội.

7. Thao, H. T. (2013). Valuing default risk for assets value jump processes. East-West Journal of Mathematics, 15(2), 101-106.

8. Thao, H. T., \& Hoang, V. Q. (2015). A Merton model of credit risk with jumps. Journal of Statistics Applications \& Probability Letters, 2(2), 97-103.

8. Thắng, Đ. H. (2013). Xác suất nâng cao. NXB Đại học Quốc gia, Hà Nội.

9. Baxter, M., \& Renie, A. (2000). Financial calculus. Cambridge University Press.

10. Etheridge, A. (2002). A course in financial calculus. Cambridge University Press.

11. Strasser, H. (2006). Introduction to probability theory and stochastic processes. Vienna Graduate School of Finance. 